\title{
Sharp constants for composition with a bi-Lipschitz measure-preserving map
}

\author{
FrédÉRIC BERNICOT AND SAHBI KERAANI
}

\begin{abstract}
In this note, we aim to describe sharp constants for the composition operator with a bi-Lipschitz measure-preserving map in several functional spaces (BMO, Hardy space, Carleson measures, etc.). It is interesting to see how the measure preserving property allows us to improve these constants. Moreover, we will prove the optimality of our results for the BMO space and describe improved estimates for solutions of transport PDEs.
\end{abstract}

Denote by $\mathcal{L}:=\mathcal{L}\left(\mathbb{R}^{d}\right)$ the group of all bi-Lipschitz homeomorphisms of $\mathbb{R}^{d}$ (equipped with the composition law $\circ$ ) and for every $\phi \in \mathcal{L}$ let

$$
K(\phi)=K_{\phi}:=\sup _{x \neq y} \frac{|\phi(x)-\phi(y)|}{|x-y|}+\frac{|x-y|}{|\phi(x)-\phi(y)|} .
$$

An easy computation yields that $K(\phi) \geq 2$, with equality if and only if $\phi$ is an isometry of $\mathbb{R}^{d}$, and moreover this quantity is sub-multiplicative: for $\phi, \psi \in \mathcal{L}$ then

$$
K(\phi \circ \psi) \leq K(\phi) K(\psi)
$$

Consequently, $\phi \mapsto \log (K(\phi))$ is a pseudo-norm on $\mathcal{L} / E(d)$, where $E(d)$ is the set of isometries on $\mathbb{R}^{d}$ to $\mathbb{R}^{d}$, which enables us to consider the group $\mathcal{L} / E(d)$ as a topological object.

2000 Mathematics Subject Classification. 42B35; 42B25.

Key words and phrases. BMO space, Composition, Measure-preserving map

The two authors are supported by the ANR under the project AFoMEN no. 2011-JS01-001-01. 
A natural question then arises: for which functional Banach space $X$ (that is, a space of functions from $\mathbb{R}^{d}$ to $\mathbb{R}$ ), is the bilinear map

$$
(f, \phi) \rightarrow f \circ \phi
$$

bounded from $X \times(\mathcal{L} / E(d))$ to $X$ ? By boundedness, we mean that we have the estimate

$$
\|f \circ \phi\|_{X} \lesssim \log (K(\phi))\|f\|_{X}
$$

We shall answer this question when $\phi \in \mathcal{L}$ is measure preserving and $X$ is the space of functions of bounded mean oscillation BMO (Section 2.1 provides the precise definition of this space). We also consider the space of Carleson measures (Section 2.3) and their dual spaces.

For a measure preserving $\phi \in \mathcal{L}$, the bi-Lipschitz property implies that for a ball $B, \phi(B)$ can be contained in a ball of radius $K_{\phi} r_{B}$, where $r_{B}$ is the radius of $B$. As a consequence, $\phi(B)$ can be covered by a collection of $K_{\phi}^{d}$ balls, with same radius as $B$. This observation easily yields that for a $B M O_{p}$ function (the BMO space with $L^{p}$ oscillation) $f$ on $\mathbb{R}^{d}$

$$
\|f \circ \phi\|_{B M O_{p}} \leq K_{\phi}^{1+d / p}\|f\|_{B M O_{p}}
$$

and since, by the John-Nirenberg property, $B M O_{p}$ coincides with BMO, by varying $p \in(1, \infty)$, if follows that for all $\epsilon>0$ we have

$$
\|f \circ \phi\|_{\mathrm{BMO}} \lesssim \epsilon K_{\phi}^{1+\epsilon}\|f\|_{\mathrm{BMO}}
$$

Let us point out that without any assumption on the map $\phi$, this inequality is optimal up to the loss $\epsilon>0$. Indeed, on $\mathbb{R}$ choose the BMO-function $f:=$ $x \mapsto \log (|x|)$ and a map $\phi$ such that $\phi(x)=x^{n}$ for $x \in[0,1]$ with $K_{\phi} \lesssim n$. Then $f \circ \phi(x)=n \log (|x|)$ for every $x \in(0,1]$ hence,

$$
K_{\phi} \lesssim n \lesssim\|f \circ \phi\|_{\mathrm{BMO}}
$$

which proves the reverse inequality and so the optimality of (1) (up to the loss $\epsilon)$.

The interesting point of this work is to prove that the dependence on the constant $K_{\phi}$ can be improved, under the measure preservation of $\phi$. In fact, the optimal dependence on $K_{\phi}$ is logarithmic. 
Sharp constants for composition with a measure-preserving map 939

Similarly, it follows that for a Carleson measure $\mu$ on $\mathbb{R}^{d}$, the pull-back measure

$$
\mu^{\sharp \phi}=\mu\left(I d \otimes \phi^{-1}\right)
$$

is a Carleson measure with

$$
\left\|\mu^{\sharp \phi}\right\|_{\mathcal{C}} \lesssim K_{\phi}^{d}\|\mu\|_{\mathcal{C}}
$$

This can also be improved to a logarithmic dependence on $K_{\phi}$ in certain circumstances.

Our main results are summarized in the following theorem.

Theorem 1. Let us assume that $\phi$ bi-Lipschitz function preserves the Lebesgue measure on $\mathbb{R}^{d}$, then

- [BMO FUNCTIONS] there exists an implicit constant (independent of $K_{\phi}$ ) such that for every BMO function $f$

$$
\|f \circ \phi\|_{\mathrm{BMO}} \lesssim \log \left(K_{\phi}\right)\|f\|_{\mathrm{BMO}}
$$

- [CARLESON MEASURES] there exist a class SC of Carleson measures and an implicit constant (independent of $K_{\phi}$ ) such that for every Carleson measure $\mu \in \mathcal{S C}, \mu^{\sharp \phi}$ belongs to $\mathcal{S} C$ and

$$
\left\|\mu^{\sharp \phi}\right\|_{\mathcal{C}} \lesssim \log \left(K_{\phi}\right)\|\mu\|_{\mathcal{C}} .
$$

Moreover, the logarithmic growth is optimal for the estimates involving the BMO norms.

Remark 1. In the light of the John-Nirenberg inequality, the result is valid for the $B M O_{p}$-norm, $p \in[1, \infty)$.

As a corollary and using $H^{1}-$ BMO duality, we have

Corollary 1. Let us assume that $\phi$ is a bi-Lipschitz function preserving the measure on $\mathbb{R}^{d}$, then there exists an implicit constant (independent of $K_{\phi}$ ) such that for every function belonging to the Hardy space $f \in H^{1}$

$$
\|f \circ \phi\|_{H^{1}} \lesssim \log \left(K_{\phi}\right)\|f\|_{H^{1}}
$$

That means in some sense that the image of an atom by the composition $a \circ \phi$ can be split into the sum of $\log \left(K_{\phi}\right)$ atoms. 
One of the main motivations of this work is the study of transport PDEs, associated with a free-divergence vector field. Indeed, such a vector field gives rise to a bi-Lipschitz measure preserving flow, which plays a crucial role for solving the transport equation. We also describe some consequences (we obtain an improved growth of the solution) in the last section for such PDEs. We point out that such study have already been done for Besov spaces, see [4, Theorem 4.2] where Vishik obtained a logarithmic growth (as our result for BMO space) for the Besov space $B_{\infty, 1}^{0}$ with applications to Euler equation. More recently, the authors have used similar ideas in [1] to get well-posedness results for Euler equation, with a vorticity belonging to a space strictly imbricated between $L^{\infty}$ and BMO. In these two results, the spaces are of completely different nature but the same idea is to understand and to have sharp inequalities for the composition (by a measure-preserving map) in these spaces.

\section{A geometric lemma}

Before proving Theorem 1, we would like to point out the key argument: a geometric lemma, which describes how a ball is modified by a measurepreserving map.

Lemma 1. For every ball $B=B\left(x_{0}, r\right)$ in $\mathbb{R}^{d}$, there exists a collection $\left(O_{k}\right)_{k}$ of balls such that:

- The collection $\left(2 O_{k}\right)_{k}$ is a bounded covering of $\phi(B)$.

- The collection $\left(O_{k}\right)_{k}$ is disjoint.

- By writing $r_{k}$ the radius of $O_{k}$ we have

$$
\frac{1}{|B|} \sum_{k}\left|O_{k}\right| \log \left(r / r_{k}\right) \lesssim \log \left(K_{\phi}\right),
$$

with an implicit constant dependent only on the dimension $n$.

Proof. Let us consider a Whitney covering of the open set $\phi(B)$ : that is a collection of open balls $\left(O_{k}\right)_{k}$ such that:

- the collection of double balls is a bounded covering:

$$
\phi(B) \subset \cup_{k} 2 O_{k}
$$

- the collection is disjoint and for all $k, O_{k} \subset \phi(B)$; 
Sharp constants for composition with a measure-preserving map 941

- the Whitney property is satisfied:

$$
r_{k} \simeq d\left(O_{k}, \phi(B)^{c}\right)
$$

So it remains for us to check (3). Indeed, this is a combinatorial argument.

First, since $\phi$ is measure preserving, it follows that $\left|O_{k}\right| \leq|B|$ and so $r_{k} \leq r$ for all $k$. For a nonnegative integer $j \geq 1$, we write

$$
u_{j}:=\sum_{k, 2^{-j} r \leq r_{k}<2^{-j+1} r}\left|O_{k}\right| .
$$

Since $\left(2 O_{k}\right)_{k}$ is a bounded covering of $\phi(B)$ (and that the balls $\left(O_{k}\right)$ are disjoint), we have

$$
\sum_{l} u_{j} \simeq\left|\phi^{-1}(B)\right|=|B|
$$

Moreover, we see that

$$
(*):=\frac{1}{|B|} \sum_{k}\left|O_{k}\right| \log \left(r / r_{k}\right) \leq \frac{1}{|B|} \sum_{j \geq 0} j u_{j} .
$$

If we denote by $C \geq 1$ the implicit constant appearing in (4), then

$$
u_{j} \leq\left|\left\{y \in \phi(B): d\left(y, \phi(B)^{c}\right) \leq C 2^{-j} r\right\}\right| .
$$

The preservation of Lebesgue measure by $\phi$ yields

$$
\left|\left\{y \in \phi(B): d\left(y, \phi(B)^{c}\right) \leq C 2^{-j} r\right\}\right|=\left|\left\{x \in B: d\left(\phi(x), \phi(B)^{c}\right) \leq C 2^{-j} r\right\}\right|,
$$

Since $\phi(B)^{c}=\phi\left(B^{c}\right)$ then

$$
u_{j} \leq\left|\left\{x \in B: d\left(\phi(x), \phi\left(B^{c}\right)\right) \leq C 2^{-j} r\right\}\right| .
$$

We set

$$
D_{j}=\left\{x \in B: d\left(\phi(x), \phi\left(B^{c}\right)\right) \leq C 2^{-j} r\right\} .
$$

Since $\phi(\partial B)=\partial[\phi(B)]$ and $\phi^{-1}$ is Lipschitz

$$
D_{j} \subset\left\{x \in B: \exists y \in \partial B:|x-y| \leq C\left\|\phi^{-1}\right\|_{L i p} 2^{-j} r \leq C K_{\phi} 2^{-j} r\right\} .
$$

Thus,

$$
u_{j} \leq\left|D_{j}\right| \lesssim K_{\phi} 2^{-j} r^{d}
$$


Coming back to $(*)$. Let $N$ be a large integer to be chosen later. We split the sum in the right-hand side into two parts

$$
(*) \lesssim \sum_{k \leq N}(\ldots)+\sum_{k>N}(\ldots . .):=I+I I .
$$

Using (5) we estimate

$$
I \leq N
$$

On the other hand, using (6),

$$
I I \lesssim \sum_{k>N} K_{\phi} j 2^{-j} \lesssim K_{\phi} N 2^{-N}
$$

This yields

$$
(*) \lesssim N+K_{\phi} N 2^{-N}
$$

Taking $N=\left[\log \left(K_{\phi}\right)\right]+1$ gives

$$
(*) \lesssim \log \left(K_{\phi}\right)
$$

as claimed.

\section{The behavior of BMO functions and Carleson measures}

\subsection{BMO functions}

For $p \in[1, \infty)$ and a locally integrable function $f$ set

$$
\|f\|_{B M O_{p}}=\sup _{B}\left(\frac{1}{|B|} \int_{B}\left|f(y)-\operatorname{Avg}_{B}(f)\right|^{p} d y\right)^{1 / p} .
$$

The John-Nirenberg property shows us that all the spaces $B M O_{p}(1 \leq p<\infty)$ coincide, so we write $B M O_{1}=B M O$.

The following lemma is classical. For sake of completeness, we will provide the proof.

Lemma 2. Let $f \in B M O$ and $B$ be a ball in $\mathbb{R}^{d}$, which contains $x \in \mathbb{R}^{d}$, then for all $\lambda>1$

$$
\left|\operatorname{Avg}_{B}(f)-\operatorname{Avg}_{\lambda B}(f)\right| \lesssim \log (\lambda)\|f\|_{\mathrm{BMO}}
$$


Sharp constants for composition with a measure-preserving map 943

Proof of Lemma 2. First we remark that, using the doubling property of the Euclidean measure,

$$
\begin{aligned}
\left|\operatorname{Avg}_{B}(f)-\operatorname{Avg}_{2 B}(f)\right| & \leq \operatorname{Avg}_{B}\left|f-\operatorname{Avg}_{2 B}(f)\right| \lesssim \operatorname{Avg}_{2 B}\left|f-\operatorname{Avg}_{2 B}(f)\right| \\
& \lesssim \operatorname{Avg}_{2 B}\left|f-\operatorname{Avg}_{2 B}(f)\right|
\end{aligned}
$$

So it follows that

$$
\left|\operatorname{Avg}_{B}(f)-\operatorname{Avg}_{2 B}(f)\right| \lesssim\|f\|_{\mathrm{BMO}}
$$

which corresponds to the desired result for $\lambda=2$.

We iterate this argument $k_{0}$ times, where $k_{0}$ is such that $2^{k_{0}} \leq \lambda<2^{k_{0}+1}$, and obtain

$$
\begin{aligned}
\left|\operatorname{Avg}_{B}(f)-\operatorname{Avg}_{2^{k_{0}} B}(f)\right| & \leq \sum_{k=0}^{k_{0}-1}\left|\operatorname{Avg}_{2^{k} B}(f)-\operatorname{Avg}_{2^{k+1} B}(f)\right| \\
& \lesssim \sum_{k=0}^{k_{0}-1}\|f\|_{\text {BMO }} \lesssim \log \left(2^{k_{0}}\right)\|f\|_{\text {BMO }}
\end{aligned}
$$

To conclude, it remains for us to estimate the following term:

$$
\begin{aligned}
\left|\operatorname{Avg}_{2^{k_{0}} B}(f)-\operatorname{Avg}_{\lambda B}(f)\right| & \lesssim \operatorname{Avg}_{2^{k_{0} B}}\left|f-\operatorname{Avg}_{\lambda B}(f)\right| \\
& \lesssim \operatorname{Avg}_{\lambda B}\left|f-\operatorname{Avg}_{\lambda B}(f)\right| \\
& \lesssim\|f\|_{\mathrm{BMO}} \lesssim \log (\lambda)\|f\|_{\mathrm{BMO}}
\end{aligned}
$$

where we have one more time used the doubling property.

We can now prove the first part of Theorem 1 .

Fix $x \in \mathbb{R}^{d}$ and let $B$ be a ball in $\mathbb{R}^{d}$ containing $x$. We wish to estimate

$$
I:=\operatorname{Avg}_{B}\left|f \circ \phi-\operatorname{Avg}_{B}(f \circ \phi)\right|
$$

and with this in mind, using the measure-preserving property of $\phi$, we see that

$$
I=\frac{1}{|B|} \int_{\phi(B)}\left|f(y)-\operatorname{Avg}_{\phi(B)}(f)\right| d y .
$$

Now we would like to compare $\phi(B)$ with the ball

$$
\tilde{B}:=B(\phi(x), r)
$$


We claim that we have the following inequality

$$
I I:=\operatorname{Avg}_{\phi(B)}\left|f-\operatorname{Avg}_{\tilde{B}}(f)\right| \lesssim \log \left(K_{\phi}\right)\|f\|_{\text {BMO }}
$$

Let us first deduce the first part of Theorem 1 from (8).

We have

$$
\left|\operatorname{Avg}_{\phi(B)}(f)-\operatorname{Avg}_{\tilde{B}}(f)\right| \lesssim \log \left(K_{\phi}\right)\|f\|_{\mathrm{BMO}}
$$

hence

$$
I \lesssim \log \left(K_{\phi}\right)\|f\|_{\mathrm{BMO}}
$$

where we have used (8) a second time and so we deduce the desired result.

It remains for us to prove (8). To achieve this we use the collection $\left(O_{k}\right)_{k}$ given by Lemma 1 . This yields

$$
I I \leq \frac{1}{|B|} \sum_{k} \int_{2 O_{k}}\left|f(y)-\operatorname{Avg}_{\tilde{B}}(f)\right| d y .
$$

Let us first remark that since $\phi$ is measure preserving then $\left|O_{k}\right| \leq|\phi(B)|=$ $|B|$ so $r_{k} \leq r$. As a consequence, we see that $2 O_{k} \subset 2 K_{\phi} \tilde{B}$ and so

$$
\begin{aligned}
\left|\operatorname{Avg}_{2 O_{k}}(f)-\operatorname{Avg}_{\tilde{B}}(f)\right| \leq & \left|\operatorname{Avg}_{2 O_{k}}(f)-\operatorname{Avg}_{2 K_{\phi} \tilde{B}}(f)\right| \\
& +\left|\operatorname{Avg}_{2 K_{\phi} \tilde{B}}(f)-\operatorname{Avg}_{\tilde{B}}(f)\right| \\
\lesssim & \left|\operatorname{Avg}_{2 O_{k}}(f)-\operatorname{Avg}_{2 K_{\phi} \tilde{B}}(f)\right|+\log \left(2 K_{\phi}\right)\|f\|_{\mathrm{BMO}} \\
\lesssim & \left|\operatorname{Avg}_{2 O_{k}}\left(f-\operatorname{Avg}_{2 K_{\phi} \tilde{B}}(f)\right)\right| \\
& +\log \left(2 K_{\phi}\right)\|f\|_{\mathrm{BMO}} \\
\lesssim & \left|\operatorname{Avg}_{B\left(x_{O_{k}}, 4 K_{\phi} r\right)}\left(f-\operatorname{Avg}_{2 O_{k}}(f)\right)\right| \\
& +\log \left(2 K_{\phi}\right)\|f\|_{\mathrm{BMO}} \\
\lesssim & \left|\operatorname{Avg}_{B\left(x_{O_{k}}, 4 K_{\phi} r\right)} f-\operatorname{Avg}_{2 O_{k}}(f)\right| \\
& +\log \left(2 K_{\phi}\right)\|f\|_{\mathrm{BMO}} \\
\lesssim & {\left[\log \left(2 K_{\phi} r / r_{k}\right)+\log \left(2 K_{\phi}\right)\right]\|f\|_{\mathrm{BMO}} } \\
\lesssim & {\left[\log \left(r / r_{k}\right)+\log \left(K_{\phi}\right)\right]\|f\|_{\mathrm{BMO}} } \\
&
\end{aligned}
$$

where we have used Lemma 2 once and then a second time with the fact that $B\left(x_{O_{k}}, 4 K_{\phi} r\right)$ is a dilation of $2 O_{k}$ by a factor $2 K_{\phi} r / r_{k}$. Consequently (using that $\left(2 O_{k}\right)_{k}$ is a bounded covering of $\phi(B)$ and that the measure is 
Sharp constants for composition with a measure-preserving map 945

preserved), we deduce that

$$
\begin{aligned}
I I & \lesssim\left(\frac{1}{|B|} \sum_{k}\left[\int_{2 O_{k}}\left|f-\operatorname{Avg}_{2 O_{k}}(f)\right|+\left|O_{k}\right|\left(\left[\log \left(r / r_{k}\right)+\log \left(K_{\phi}\right)\right]\|f\|_{\mathrm{BMO}}\right)\right]\right) \\
& \lesssim\left(\frac{1}{|B|} \sum_{k}\left|O_{k}\right|\left(\log \left(r / r_{k}\right)+\log \left(K_{\phi}\right)\right)\right)\|f\|_{\mathrm{BMO}} \\
& \lesssim \log \left(K_{\phi}\right) \mid f\left\|_{\mathrm{BMO}}+\left(|B|^{-1}\left[\sum_{k}\left|O_{k}\right| \log \left(r / r_{k}\right)\right]\right)\right\| f \|_{\mathrm{BMO}}
\end{aligned}
$$

where we have used that $\sum_{k}\left|O_{k}\right| \simeq|B|$. The proof is also achieved by invoking property (3) to compute the remaining sum and estimate it by the expected quantity $\log \left(K_{\phi}\right)\|f\|_{\text {BMO }}$.

\subsection{Optimality of the logarithmic growth}

We begin by observing that if $\phi$ is a measure preserving bi-Lipschitz function then the Lipschitz norms of $\phi$ and $\phi^{-1}$ are logarithmically equivalent.

Lemma 3. There exists $C=C(d)$ such that for every smooth measure preserving $\phi \in \mathcal{L}$

$$
\frac{1}{C}\|\phi\|_{\text {Lip }}^{\frac{1}{d-1}} \leq\left\|\phi^{-1}\right\|_{\text {Lip }} \leq C\|\phi\|_{\text {Lip }}^{d-1} .
$$

In particular, if $\|\phi\|_{\text {Lip }}$ is large enough,

$$
\log \left(K_{\phi}\right) \lesssim \log \left(\|\phi\|_{\text {Lip }}\right)
$$

Proof. By symmetry it is enough the prove the second inequality only. Since $\phi$ is a measure preserving map then

$$
|\operatorname{det}(D \phi(x))|=1, \quad \forall x \in \mathbb{R}^{d} .
$$

Take $x \in \mathbb{R}^{d}$ and denote $y=\phi^{-1}(x)$. Since $D\left(\phi^{-1}\right)(x)=(D \phi(y))^{-1}$ then

$$
\left\|D\left(\phi^{-1}\right)(x)\right\| \lesssim \max _{\lambda \in \Sigma(y)}|\lambda|^{-1} \leq\left[\max _{\lambda \in \Sigma(y)}|\lambda|\right]^{d-1} \lesssim\|D \phi(y)\|^{d-1},
$$

where $\Sigma(y)$ is the spectrum of $\sqrt{D \phi(y)^{*} D \phi(y)}$. The second inequality is due to the fact that $\prod_{\lambda \in \Sigma(y)} \lambda=1$. 
Finally, we obtain

$$
\left\|D \phi^{-1}\right\|_{L^{\infty}} \lesssim\|D \phi\|_{L^{\infty}}^{d-1}
$$

as claimed.

Our argument for the optimality of the logarithmic growth relies on properties of quasi-conformal mappings. Given that we can restrict our attention to bi-Lipschitz functions, we can take as our definition of $\phi \in \mathcal{L}$ being $K$-quasi-conformal that it satisfies the inequality

$$
\|D \phi(x)\|_{L^{\infty}}^{d} \leq K \operatorname{det}(D \phi)(x)
$$

for all $x \in \mathbb{R}^{d}$. If $\phi$ is also measure preserving, then this inequality reduces to

$$
\|D \phi\|_{L^{\infty}}^{d} \leq K
$$

so in this context, $K$-quasiconformality is simply that the size of the Lipschitz constant of $\phi$ is bounded by $K^{1 / d}$. We will use the following theorem by H.M. Riemann [3, Theorem 3].

Theorem 2. Assume that $\phi \in \mathcal{L}$ is orientation preserving. If the induced map $f \mapsto f \circ \phi$ is a bijective isomorphism of BMO and satisfies

$$
\|f \circ \phi\|_{B M O} \leq \kappa\|f\|_{B M O}
$$

for all $f \in B M O$ then $\phi$ is a K-quasiconformal mapping with $K=e^{(d-1)(C \kappa-1)}$, for a fixed $C>0$ depending only on the dimension $d$.

If in the context of this theorem one also assumes that $\phi$ satisfies (9) (which is the case if $\phi$ preserves the Lebesgue measure) then

$$
C_{d} \log \left(\|D \phi\|_{L^{\infty}}\right) \leq \kappa
$$

Combined with Lemma 3 this proves the optimality of the logarithmic growth in Theorem 1. 
Sharp constants for composition with a measure-preserving map 947

\subsection{The behavior of some Carleson measures}

Let $\mu$ be a Carleson measure on $\mathbb{R}^{+} \times \mathbb{R}^{d}$. We recall that this means that

$$
\|\mu\|_{\mathcal{C}}:=\sup _{\text {ball } B \subset \mathbb{R}^{d}}|B|^{-1} \mu(T(B))<\infty
$$

where $T(B)$ is the Carleson box over the ball $B$ defined by

$$
T(B):=\{(x, t), x \in B, 0<t \leq r\}=B \times(0, r] .
$$

Definition 1. Let $\mu$ be a Carleson measure and $\phi$ a bi-Lipschitz measurepreserving map on $\mathbb{R}^{d}$. We denote $\mu^{\sharp \phi}$ the pull-back measure, defined by

$$
\mu^{\sharp \phi}(I \times A)=\mu\left(I \times \phi^{-1}(A)\right),
$$

for every time interval $I$ and measurable set $A \subset \mathbb{R}^{d}$.

Definition 2. Let $\beta$ be a measurable map from $\mathbb{R}^{+} \times \mathbb{R}^{d} \rightarrow \mathbb{R}$ and we define the measure

$$
\mu:=\mu_{\beta}=|\beta(t, x)|^{2} \frac{d t d x}{t} .
$$

Consequently, we infer

$$
d \mu^{\sharp \phi}(t, x)=|\beta(t, \phi(x))|^{2} \frac{d t d x}{t}
$$

so $\mu^{\sharp \phi}=\mu_{\beta^{\phi}}$ with $\beta^{\phi}(t, x)=\beta(t, \phi(x))$.

Theorem 3. Let $\phi$ a bi-Lipschitz measure-preserving map on $\mathbb{R}^{d}$ and $\mu=\mu_{\beta}$ be a Carleson measure associated to some $\beta \in L^{\infty}\left(\mathbb{R}^{+} \times \mathbb{R}^{d}\right)$. Then there exists an implicit constant (only dependent on $n$ ) such that $\mu^{\sharp \phi}$ is a Carleson measure with

$$
\left\|\mu^{\sharp \phi}\right\|_{\mathcal{C}} \lesssim\|\mu\|_{\mathcal{C}}+\log \left(K_{\phi}\right)\|\beta\|_{L^{\infty}\left(\mathbb{R}^{+} \times \mathbb{R}^{d}\right)}^{2} .
$$

Proof. Let consider $B=B\left(x_{0}, r\right)$ a ball of $\mathbb{R}^{d}$ and its Carleson box $T(B)$. We have to estimate

$$
\begin{aligned}
\mu^{\sharp \phi}(T(B)) & =\int_{[0, r] \times B}|\beta(t, \phi(x))|^{2} \frac{d t d x}{t} \\
& =\int_{[0, r] \times \phi(B)}|\beta(t, x)|^{2} \frac{d t d x}{t} .
\end{aligned}
$$


Aiming that, we use the collection $\left(O_{k}\right)$ given by Lemma 1 to cover $\phi(B)$ (with $p=1$ ). Then (we remember that as previously we have $r_{k} \leq r$ ), it follows:

$$
\begin{aligned}
\mu^{\sharp \phi}(T(B))= & \sum_{k} \int_{[0, r] \times 2 O_{k}}|\beta(t, x)|^{2} \frac{d t d x}{t} \\
\leq & \sum_{k} \int_{\left[0, r_{k}\right] \times 2 O_{k}}|\beta(t, x)|^{2} \frac{d t d x}{t} \\
& +\sum_{k} \int_{\left[r_{k}, r\right] \times 2 O_{k}}|\beta(t, x)|^{2} \frac{d t d x}{t} \\
\leq & \sum_{k} \mu\left(T\left(2 O_{k}\right)\right)+\|\beta\|_{L^{\infty}\left(\mathbb{R}^{+} \times \mathbb{R}^{d}\right)}^{2} \sum_{k}\left|O_{k}\right| \log \left(\frac{r}{r_{k}}\right) \\
\lesssim & \|\mu\|_{\mathcal{C}}\left(\sum_{k}\left|O_{k}\right|\right)+\|\beta\|_{L^{\infty}\left(\mathbb{R}^{+} \times \mathbb{R}^{d}\right)}^{2} \log \left(K_{\phi}\right)|B| \\
\lesssim & \left(\|\mu\|_{\mathcal{C}}+\log \left(K_{\phi}\right)\|\beta\|_{L^{\infty}\left(\mathbb{R}^{+} \times \mathbb{R}^{d}\right)}^{2}\right)|B|,
\end{aligned}
$$

where we used the doubling property of the Euclidean measure, the disjointness of the balls $\left(O_{k}\right)_{k}$ and the property (3). The proof is also concluded.

Corollary 2. Let define $S C$ the class of Carleson measure $d \mu=|\beta(t, x)|^{2} \frac{d t d x}{t} \quad$ satisfying $\|\beta\|_{L^{\infty}} \lesssim\|\mu\|_{\mathcal{C}}$, equipped with the norm $\|\mu\|_{\mathcal{S C}}:=\|\mu\|_{\mathcal{C}}$. Then we have

$$
\left\|\mu^{\sharp \phi}\right\|_{\mathcal{S} C} \lesssim \log \left(K_{\phi}\right)\|\mu\|_{\mathcal{S} C} .
$$

Example. We know that for some standard "approximations of unity" kernels $\left(K_{t}\right)$, for a $L_{l o c}^{1}$ function $g$ we can build the measure

$$
d \mu_{g}(t, x)=\left|\int K_{t}(x, y) g(y) d y\right|^{2} \frac{d t d x}{t}
$$

Then it is well known that $d \mu_{g}$ is a Carleson measure if and only if $g \in B M O$ (see [2]). Moreover, it is easy to check that such measures belong to $\mathcal{S C}$. 
Sharp constants for composition with a measure-preserving map 949

\section{Applications to some PDEs}

\subsection{The Transport equation by a free-divergence vector field}

Let $v: \mathbb{R}^{d} \rightarrow \mathbb{R}^{d}$ be a divergence free ${ }^{1}$ Lipschitz vector field and consider the transport equation:

$$
\left\{\begin{array}{l}
\partial_{t} u-v \cdot \nabla(u)=0 \\
u_{\mid t=0}=u_{0}
\end{array}\right.
$$

with an initial data $u_{0}$. Then it is well known that a smooth solution is constant along the characteristics given by the vector field. Indeed, consider the flow $\phi: \mathbb{R}_{+} \times \mathbb{R}^{d} \rightarrow \mathbb{R}^{d}$, solution of

$$
\left\{\begin{array}{l}
\partial_{t} \phi=v(\phi), \\
\phi(0, x)=x,
\end{array}\right.
$$

then the divergence free assumption on $v$ yields that $\phi(t, \cdot)$ is a Lebesgue measure preserving diffeomorphism, for every $t \in \mathbb{R}$. Moreover, any smooth solution $u$ of the transport equation is unique and is given by

$$
u(t, x)=u_{0}\left(\phi_{t}^{-1}(x)\right) .
$$

It is well known using Gronwall lemma that

$$
K(\phi(t, \cdot)) \lesssim e^{\|v\|_{\text {Lip }} t}
$$

where $\|v\|_{\text {Lip }}$ is the Lipschitz constant of the vector field.

As a consequence, the previous Theorem and Corollary imply the following:

Theorem 4. Let $u$ be the unique solution of (11).

1) If $u_{0} \in B M O$, then $u \in L_{l o c}^{\infty}(B M O)$ and

$$
\|u(t)\|_{\mathrm{BMO}} \lesssim\left[1+\|v\|_{L i p} t\right]\left\|u_{0}\right\|_{\mathrm{BMO}}, \quad \forall t \geq 0
$$

2) If $u_{0} \in \operatorname{Lip}_{p}(a) \quad$ (for some $a \in(0,1]$ and $p \in(1, \infty)$ ), then $u \in L_{l o c}^{\infty}\left(\operatorname{Lip}_{p}(a)\right)$ and

$$
\|u(t)\|_{\operatorname{Lip}_{p}(a)} \lesssim e^{a\|v\|_{L i p} t}\left\|u_{0}\right\|_{L_{\text {Lip }}(a)}, \quad \forall t \geq 0 .
$$

\footnotetext{
${ }^{1}$ That means $\nabla \cdot v=0$.
} 


\subsection{The perturbed transport equation}

Consider the following transport equation with a linear Riesz-type second member term:

$$
\left\{\begin{array}{l}
\partial_{t} \omega+(u \cdot \nabla) \omega=\mathcal{R} \omega \\
\omega_{\mid t=0}=\omega_{0}
\end{array}\right.
$$

where $\mathcal{R}$ is a Riesz operator. This type of equation naturally arises when one considers for example the perturbed two-dimensional (2D) Euler equations which is obtained by adding a zero order term to the incompressible $2 \mathrm{D}$ Euler system. Let us for example consider the following system

$$
\left\{\begin{array}{l}
\partial_{t} u+(u \cdot \nabla) u=-\nabla p+A u \\
\operatorname{div}(u)=0 \\
u_{\mid t=0}=u_{0}
\end{array}\right.
$$

with $A u=\left(u^{1}, 0\right)$. Then the vorticity $\omega:=\operatorname{curl}(u)=\partial_{1} u^{2}-\partial_{2} u^{1}$ satisfies the following equation

$$
\partial_{t} \omega+u \cdot \nabla \omega=\partial_{22} \Delta^{-1} \omega
$$

The continuity of Riesz operator on $L^{p}$ for every $1<p<\infty$, the divergence free condition and Gronwall inequality imply together

$$
\|\omega(t)\|_{L^{p}} \leq\left\|\omega_{0}\right\|_{L^{p}} e^{C_{p} t}, \quad \forall t \geq 0
$$

Here $C_{p}=\|\mathcal{R}\|_{\mathcal{L}\left(L^{p}, L^{p}\right)} \simeq \frac{p^{2}}{p-1}$. However, it is not clear at all how one can obtain an $L^{\infty}$ estimates since the Riesz operator $\partial_{22} \Delta^{-1}$ is not continuous on that space. A natural idea is to replace the space $L^{\infty}$ by another space with similar "scaling" but stable for $\mathcal{R}$ (such that $B M O$ for example). However, in this case a problem of composition arises: an extra term depending of the Lipschitz norm of $u$ appears and the estimate is no longer closable.

Theorem 2 shows that we cannot avoid the constants generated by the composition with the flow (nor even to improve them). A bound for the $B M O$-norm similar to (14) cannot be obtained directly: indeed with the best constants, we already have a quadratic estimate which is not Gronwallisable. However, Theorem 1 can be applied in order to get sharper a priori estimates. In fact, consider $u$ be a smooth solution of (12) and the corresponding flow 
Sharp constants for composition with a measure-preserving map 951 $\phi: \mathbb{R}_{+} \times \mathbb{R}^{d} \rightarrow \mathbb{R}^{d}$, solution of

$$
\left\{\begin{array}{l}
\partial_{t} \phi=u(t, \phi) \\
\phi(0, x)=x
\end{array}\right.
$$

Hence by Gronwall Lemma and using the $B M O$-boundedness of the Riesz transform, we get

$$
\|\omega(t, \phi(t, \cdot))\|_{\mathrm{BMO}} \lesssim\left\|\omega_{0}\right\|_{\mathrm{BMO}} \exp (c t)
$$

for some numerical constant $c>0$, and so by Theorem 1

$$
\|\omega(t, \cdot)\|_{\mathrm{BMO}} \lesssim\left\|\omega_{0}\right\|_{\mathrm{BMO}}\left(1+\|u\|_{L_{t}^{1} \operatorname{Lip}}\right) \exp (c t)
$$

If the vector-field satisfies

$$
\|u\|_{L_{t}^{1} \operatorname{Lip}} \leq \exp (\alpha t)
$$

for some $\alpha>0$, then one has a similar estimate than all the $L^{p}$ norm. It is worthy of noticing that a rough estimate (involving $K(\phi)$ instead of $\log K(\phi))$ gives

$$
\|\omega(t, \cdot)\|_{\mathrm{BMO}} \lesssim\left\|\omega_{0}\right\|_{\mathrm{BMO}} \exp \left(\|u\|_{L_{t}^{1} \operatorname{Lip}}\right) \exp (c t)
$$

The merit of the next result is only the improvement of the estimate of the growth of the $B M O$ norm of the solution.

Proposition 1. Let $u$ be a divergence free vector fields and $\omega$ a smooth solution of (12). If $\omega_{0} \in B M O$, then

$$
\|\omega(t)\|_{\mathrm{BMO}} \lesssim\left\|\omega_{0}\right\|_{\mathrm{BMO}}\left(1+\|u\|_{L_{t}^{1} L i p}\right) \exp (c t)
$$

for all $t \geq 0$.

\section{Acknowledgments}

The authors are thankful to Carlos Perez for having given reference [3] to prove the optimality of the logarithmic growth and to David Rule for valuable advices to improve this paper. 


\section{References}

[1] F. Bernicot and S. Keraani, On the global wellposedness of the 2D Euler equations for a large class of Yudovich type data, Ann. Sci. Éc. Norm. Supér. 47(3) (2014), 559-576.

[2] C. Fefferman and E.M. Stein, $H^{p}$ spaces of several variables, Acta Math. 129 (1971), 137-193.

[3] H.M. Reimann, Functions of bounded mean Oscillation and Quasiconformal Mappings, Comment. Math. Helv. 49 (1974), 260-276.

[4] M. Vishik, Hydrodynamics in Besov Spaces, Arch. Rational Mech. Anal. 145 (1998), 197-214.

CNRS - UNIVERSITÉ DE NANTES

Laboratoire de Mathématiques Jean Leray

2, Rue de la Houssinière F-44322 Nantes Cedex 03

FRANCE

E-mail address: frederic.bernicot@univ-nantes.fr

UFR DE MAThÉMATIQUES

UNIVERSité DE LILLE 1

59655 Villeneuve D'Ascq Cedex

FRANCE

E-mail address: sahbi.keraani@univ-lille1.fr

Received August 12, 2012 